Florida International University FIU Digital Commons

\title{
Tests of the Intersensory Redundancy Hypothesis across Early Postnatal Development
}

Jimena Vaillant-Mekras

Florida International University, jvail001@fiu.edu

DOI: $10.25148 /$ etd.FI12080604

Follow this and additional works at: https://digitalcommons.fiu.edu/etd

\section{Recommended Citation}

Vaillant-Mekras, Jimena, "Tests of the Intersensory Redundancy Hypothesis across Early Postnatal Development" (2012). FIU Electronic Theses and Dissertations. 682.

https://digitalcommons.fiu.edu/etd/682 


\section{FLORIDA INTERNATIONAL UNIVERSITY}

Miami, Florida

\section{TESTS OF THE INTERSENSORY REDUNDANCY HYPOTHESIS ACROSS EARLY POSTNATAL DEVELOPMENT}

A dissertation submitted in partial fulfillment of

the requirements for the degree of

DOCTOR OF PHILOSOPHY

in

PSYCHOLOGY

by

Jimena Vaillant-Mekras

2012 


\section{To: Dean Kenneth Furton}

College of Arts and Sciences

This dissertation, written by Jimena Vaillant-Mekras, and entitled Tests of the Intersensory Redundancy Hypothesis Across Early Postnatal Development, having been approved in respect to style and intellectual content, is referred to you for judgment.

We have read this dissertation and recommend that it be approved.

Mary Levitt

Charles Bigger

Lorraine E. Bahrick

Robert Lickliter, Major Professor

Date of Defense: July 20, 2012

The dissertation of Jimena Vaillant-Mekras is approved.

Dean Kenneth Furton

College of Arts and Sciences

Dean Lakshmi N. Reddi

University Graduate School

Florida International University, 2010 


\section{DEDICATION}

I dedicate this dissertation to my parents and my husband, John. Their support, encouragement, patience, and love have made this achievement possible. 


\section{ACKNOWLEDGMENTS}

I wish to thank my committee members for their participation and guidance throughout this process. I would also like to thank my major professor, Dr. Robert Lickliter, for his incredible training and support throughout my graduate career. Finally, I would like to acknowledge the MBRS RISE program for providing the resources and opportunities that have enhanced my research experience. 


\title{
ABSTRACT OF THE DISSERTATION \\ TESTS OF THE INTERSENSORY REDUNDANCY HYPOTHESIS ACROSS EARLY POSTNATAL DEVELOPMENT
}

\author{
by \\ Jimena Vaillant-Mekras \\ Florida International University, 2012 \\ Miami, Florida

\section{Professor Robert Lickliter, Major Professor}

The Intersensory Redundancy Hypothesis (IRH; Bahrick \& Lickliter, 2000, 2002, 2012) predicts that early in development information presented to a single sense modality will selectively recruit attention to modality-specific properties of stimulation and facilitate learning of those properties at the expense of amodal properties (unimodal facilitation). Vaillant (2010) demonstrated that bobwhite quail chicks prenatally exposed to a maternal call alone (unimodal stimulation) are able to detect a pitch change, a modality-specific property, in subsequent postnatal testing between the familiarized call and the same call with altered pitch. In contrast, chicks prenatally exposed to a maternal call paired with a temporally synchronous light (redundant audiovisual stimulation) were unable to detect a pitch change. According to the IRH (Bahrick \& Lickliter, 2012), as development proceeds and the individual's perceptual abilities increase, the individual should detect modality-specific properties in both nonredundant, unimodal and redundant, bimodal conditions. However, when the perceiver is presented with a difficult task, relative to their level of expertise, unimodal facilitation should become evident. The first experiment of the present study exposed bobwhite quail chicks $24 \mathrm{hr}$ after hatching to unimodal 
auditory, nonredundant audiovisual, or redundant audiovisual presentations of a maternal call for $10 \mathrm{~min} / \mathrm{hr}$ for 24 hours. All chicks were subsequently tested $24 \mathrm{hr}$ after the completion of the stimulation ( $72 \mathrm{hr}$ following hatching) between the familiarized maternal call and the same call with altered pitch. Chicks from all experimental groups (unimodal, nonredundant audiovisual, and redundant audiovisual exposure) significantly preferred the familiarized call over the pitch-modified call. The second experiment exposed chicks to the same exposure conditions, but created a more difficult task by narrowing the pitch range between the two maternal calls with which they were tested. Chicks in the unimodal and nonredundant audiovisual conditions demonstrated detection of the pitch change, whereas the redundant audiovisual exposure group did not show detection of the pitch change, providing evidence of unimodal facilitation. These results are consistent with predictions of the IRH and provide further support for the effects of unimodal facilitation and the role of task difficulty across early development. 


\section{TABLE OF CONTENTS}

CHAPTER

PAGE

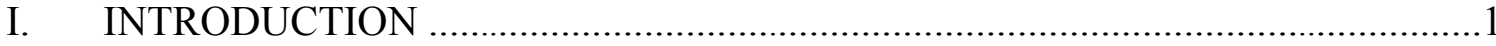

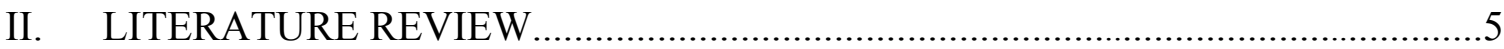

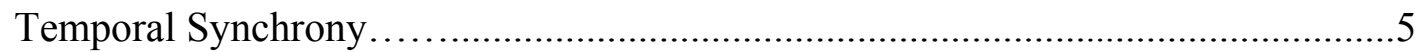

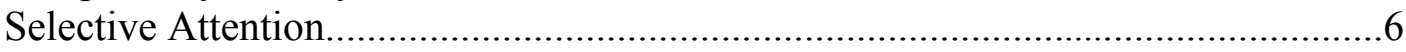

Intersensory Redundancy Hypothesis.......................................

III. DETECTION OF MODALITY-SPECIFIC PROPERTIES DURING PRENATAL

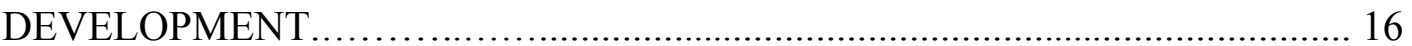

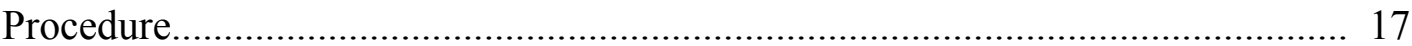

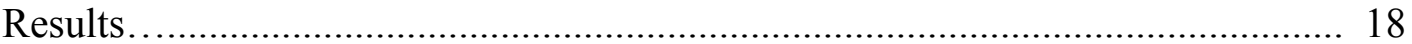

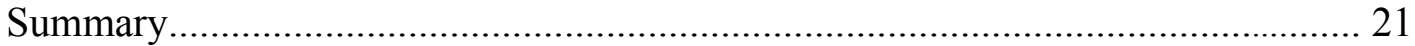

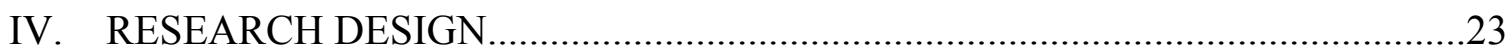

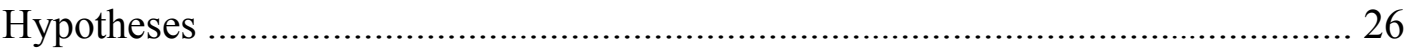

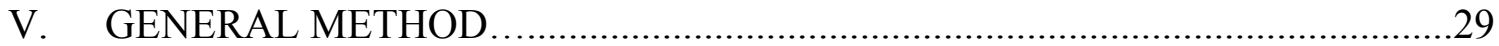

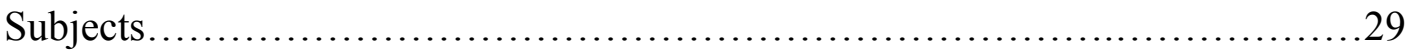

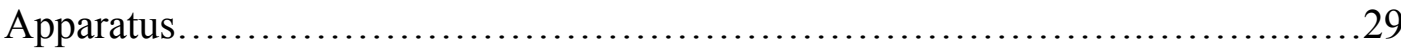

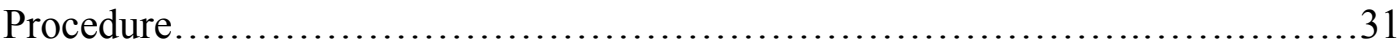

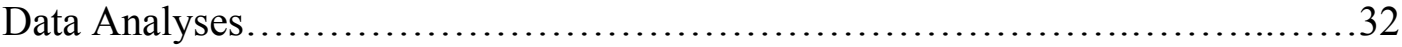

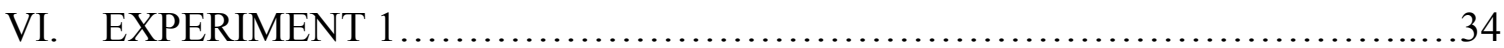

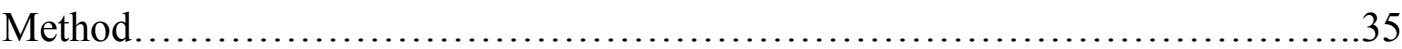

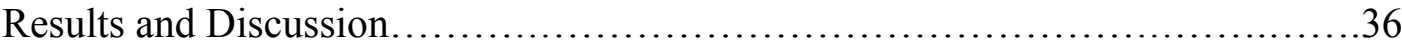

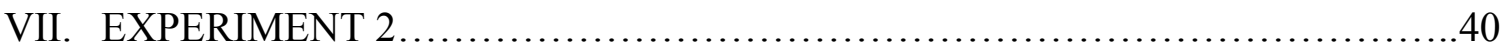

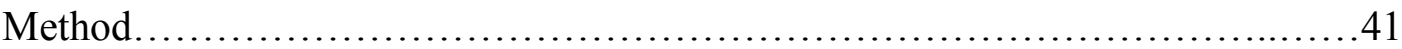

Results and Discussion................................................... 42

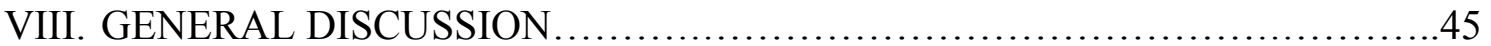

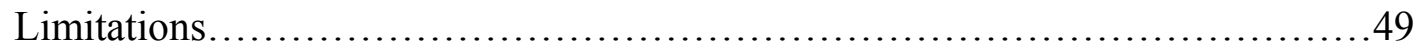

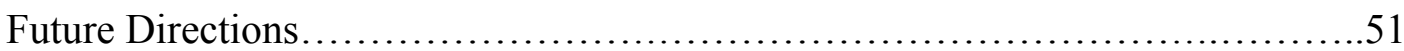

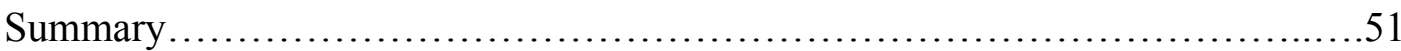

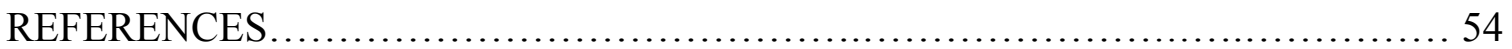

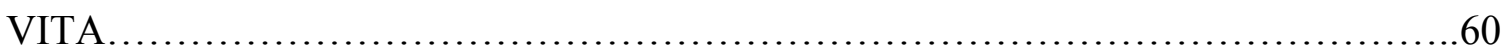




\section{LIST OF TABLES}

TABLE

PAGE

1. Means and Standard Deviations of Duration Scores (in seconds) $\ldots \ldots \ldots \ldots \ldots \ldots \ldots \ldots$

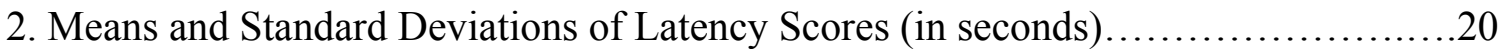

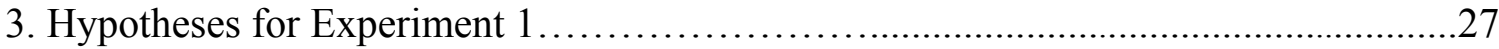

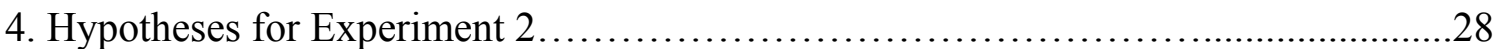

5. Means and Standard Deviations of Duration Scores (in seconds) for Experiment 1....37

6. Mean Proportion of Total Duration Time and Standard Deviations (in seconds) for the

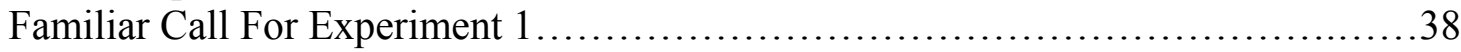

7. Means and Standard Deviations of Duration Scores (in seconds) for Experiment 2...43

8. Mean Proportion of Total Duration Time and Standard Deviations (in seconds) for the

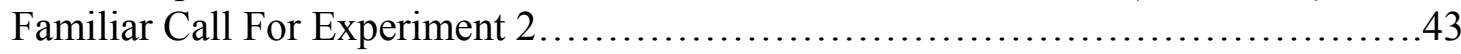




\section{CHAPTER I}

\section{INTRODUCTION}

Organisms develop in an environment rich with multimodal stimulation. Human and animal infants thus gain enormous amounts of experience with multisensory information during the postnatal period. Research has consistently shown that young infants perceive multimodal information readily in the weeks and months after birth (Bremner, Lewkowicz, \& Spence, 2012; Lewkowicz \& Lickliter, 1994; Lickliter \& Bahrick, 2000). For instance, a series of studies by Bahrick (1983, 1987, 1988, 1992) found that 3-6 month-old infants are able to match soundtracks to the appropriate object hitting a surface based on the material and composition of the object. Further, infants can match voices and faces on the basis of gender (Walker-Andrews, Bahrick, Raglioni, \& Diaz, 1991). Six-month old infants were shown videos of a male face and female face accompanied by a soundtrack of a single voice. Infants showed preferential looking to the gender-appropriate face that corresponded with the voice, indicating that they are able to match the voice with the appropriate face on the basis of gender.

As development proceeds, adults must become adept at picking up features in the environment that are particularly relevant while ignoring others in order to attend to specific events. For example, attending to dialogue in a movie while ignoring nearby conversations or avidly observing a sporting event entails picking up and attending to specific information that is pertinent to the understanding of the event. Perception serves as the foundation for selecting and interpreting the events in the world. Hence, it is critical to understand how selective attention and perception develop in infancy, thereby 
allowing individuals to ultimately make sense of the multisensory information available in their natural environments.

The rapid learning and improvement of perceptual skills over the course of infancy is the result of ongoing experience with the structure of the environment and includes more efficient allocation of attention and more efficient pick up of information. Young organisms quickly come to attend to meaningful features and variations in the environment and ignore irrelevant ones (Gibson, 1969, 1988; Gibson \& Pick, 2000). The attentional selectivity in early development is thought to be driven by the young organism's sensitivity to several salient properties of stimulation, particularly amodal properties such as intensity and redundancy (Lewkowizc \& Turkewitz, 1980; Bahrick \& Lickliter, 2002, 2012).

Research has demonstrated that detection of amodal properties of stimulation such as temporal synchrony, intensity, and tempo, are critical to early perceptual development (Bahrick \& Pickens, 1994; Bahrick \& Lickliter, 2002, Lewkowicz, 2000; Lewkowicz \& Lickliter, 1994 for reviews). Amodal properties are features of stimulation that are redundant across two or more sense modalities. For instance, time, space, and intensity can be perceived by multiple senses. Most events provide this type of information because they take place across time and space and have specific intensity patterns. Processing of properties of stimulation progresses from global to increasingly more detailed levels of stimulation. As infants develop, their ability to perceive and gain meaning from their environment improves. For example, Bahrick (1987) examined infants' intermodal perception of single and multiple objects through detection of the invariant temporal microstructure of an object. Temporal microstructure is the internal 
temporal organization of each impact sound and motion, which specifies the composition of an object (i.e., single versus compound). Three, 4, and 6-month old infants were presented with two side-by-side films of a transparent cylinder, one with a large marble and the other with several smaller marbles. The two cylinders were shaken back and forth, while a central speaker between the two screens presented the natural soundtrack of only one of the films. Although not seen with the younger infants, the 6-month olds were able to match the video with the corresponding sound based on the temporal microstructure that denotes single versus compound objects. However, using a more sensitive habituation procedure, Bahrick (2001) showed that infants as young as 4 weeks old can detect global temporal synchrony relations but not temporal microstructure that specifies object composition, a more detailed amodal property. Sensitivity to object composition emerged by 7 weeks of age. These results demonstrate the developmental trend in which older infants are better able to detect more specific levels of amodal information (in this case, temporal information) of multimodal events. This increasing sensitivity to certain properties in the environment is a cornerstone of perceptual development. What guides this developmental process in young infants?

The use of animal models to explore experiential factors that contribute to early perceptual development has provided considerable knowledge on this topic, as studies that modify sensory experience during perinatal development are difficult and often impossible to carry out with human infants (Lickliter \& Bahrick, 2000). Several studies with precocial birds have demonstrated that altering sensory experience during early development can significantly impact perceptual learning and development (Gottlieb, 1971, 1993; Lickliter \& Strombos, 1991, Honeycutt \& Lickliter, 2002). Lickliter and 
Leowkowicz (1995) suggested that there is an optimal range of prenatal sensory stimulation that is required for species-typical perceptual abilities to develop. For example, bobwhite quail embryos incubated in groups typically show auditory learning of a maternal call. However, chicks incubated in isolation during late prenatal development do not show auditory learning or respond to visual cues at ages chicks incubated in groups demonstrate species-typical auditory and visual responsiveness.

Like perceptual development, the development and deployment of selective attention is the result of ongoing experience with the properties of objects and events. Despite various human and animal studies examining perceptual abilities, more research is needed to explore the role of selective attention during early development and how unimodal and multisensory experience affects attentional selectivity and intersensory perception across development. The current study addresses these topics. 


\section{CHAPTER II}

\section{LITERATURE REVIEW}

Research has shown that amodal properties (features of stimulation that are detected through multiple sense modalities, such as tempo and rhythm) are particularly salient in early development and are also detected earlier in development than modalityspecific properties of stimulation (features of stimulation that are only detected through one sense modality alone, such as color or pitch) (Lewkowicz \& Lickliter, 1994; Gibson \& Pick, 2000; Bahrick 1992, 1994, 2001; Lewkowicz, 2000). These findings are in agreement with ecological theories of perception (Gibson, 1966, 1979). Gibson's ecological view $(1966,1979)$ proposed that the senses work in conjunction to pick up and attend to invariants in the environment in order to perceive unitary multimodal events. Hence, detecting amodal relations in the environment allows the young infant to successfully attend to and make sense of objects and events.

\section{Temporal Synchrony}

Infants are continuously presented with stimulation from all the senses. How do they decipher which sights and sounds are related and which are not? Temporal synchrony, a global amodal property, is thought to bind stimulation across the senses, allowing for the perceiver to determine which sights and sounds belong together or originate from the same event rather than associating unrelated sensory stimulation (Bahrick \& Pickens, 1994; Bahrick \& Lickliter, 2002; Lewkowicz, 2000). Several studies have shown that orientating to an audio-visual stimulus is facilitated when auditory and visual cues are temporally synchronized (Jiang, Jiang, \& Stein, 2002, Stein, Meredith, Honeycutt, \& McDade, 1989). For instance, cats' orienting response toward a visual 
stimulus is enhanced by the presentation of a temporally synchronous auditory stimulus (Stein, Meredith, Honeycutt, \& McDade, 1989). Synchrony also enhances infants' detection of changes in amodal properties. For instance, Lewkowicz $(1988,1996)$ demonstrated that 10-month old infants who had been habituated to an inanimate audiovisual stimulus did not respond to changes occurring solely in the visual component of the stimulus; similarly, 4-month old infants habituated to a video showing a speaking human face did not respond to changes occurring solely in the audible component (i.e., voice). In fact, these infants were only able to discriminate changes in amodal properties when changes in the audible and visual components occurred in synchrony. These findings indicate that temporal synchrony enhances the ability to discriminate changes in amodal properties of events.

Temporal synchrony also aids in the coordination of attention (Bahrick, Walker, \& Neisser, 1981; Lewkowicz, 2000; Lickliter \& Bahrick, 2000; Jaime, Bahrick, \& Lickliter, 2010). For example, 4-month old infants presented with two superimposed films of moving objects and an audio track that corresponded to only one of the films were able to attend specifically to the film that was in synchrony with the soundtrack while ignoring the silent superimposed film (Bahrick, Walker, \& Neisser, 1981). Jaime, Bahrick, and Lickliter (2010) showed that bobwhite quail embryos exposed to a flash of light temporally synchronized only with the first note of the call enhanced perceptual learning of the entire call compared to unimodal exposure or nonredundant bimodal (not synchronous) exposure. Hence, temporal synchrony guides selective attention during early development, providing the young organism redundant information about the cohesion and location of multimodal events. 


\section{Selective Attention}

Selective attention, the orienting and processing of specific information at the expense of other information, guides the perceptual system and allows the individual to focus, learn, and acquire meaning in the world. For example, the ability to engage effectively in conversation with another person while dismissing irrelevant noise or background information is required for appropriate social engagement and communication.

The concept of salience hierarchies (or saliency maps) has been used to explain what features of the environment are attended to and others ignored (Adler, Gerhardstein, \& Rovee-Collier, 1998; Bahrick \& Newell, 2008; Koch \& Ullman, 1985). Specifically, when attentional resources are taxed, selective attention is thought to be first directed toward highly salient features and then progressively shift toward less salient features across exploratory time. It is likely that selective attention to salient features in the environment promotes detailed processing and learning of those features and has further compounding effects on perceptual, language, and cognitive development (Bahrick \& Lickliter, 2012). Intersensory Redundancy Hypothesis

The Intersensory Redundancy Hypothesis (Bahrick \& Lickliter, 2000, 2002, 2012) describes the conditions under which selective attention and perceptual processing are directed toward specific properties of events (amodal or modality-specific) in early development. Intersensory redundancy is the temporally and spatially synchronized occurrence of the same information (e.g., duration, tempo) across two or more senses. The IRH suggests that intersensory redundancy is highly attractive to young organisms 
and guides selective attention to amodal properties of stimulation that are redundant across multiple senses at the expense of nonredundant properties of stimulation within the same event. This attentional selectivity aids in the perception, learning, and memory for amodal properties.

The IRH has four testable predictions:

1) Redundant multimodal stimulation recruits attention to and facilitates perceptual processing of amodal information of events (at the cost of nonredundant properties) more so than does unimodal (e.g. auditory or vestibular) stimulation. This process is referred to as intersensory facilitation.

2) Non-redundantly specified information (information presented to one sense modality alone) recruits attention and facilitates perceptual processing of modality-specific properties of events more so than does multimodal stimulation. This is termed unimodal facilitation, and occurs partly because there is no redundancy competing for attention in unimodal stimulation.

3) As development proceeds, attention becomes increasingly efficient, better organized, and more flexible with experience, thereby allowing for the detection of both amodal and modality-specific information in both redundant, multimodal and nonredundant, unimodal stimulation.

4) When task difficulty is high and attentional resources are limited, intersensory and unimodal facilitation occur across the lifespan. 


\section{Intersensory Facilitation}

A number of studies of both human and animal infants have provided support for these four predictions. Intersensory facilitation has been observed in contexts of social and nonsocial events and across species. For example, Flom \& Bahrick (2007) found that 4-month old infants can discriminate a change in a woman's affect (happy, sad, angry) when the woman's face and voice are presented in synchrony, but not when the face is presented only visually or when the face and voice are presented asynchronously. Further, infants are better able to perceive tempo and rhythm when they are redundant across two sense modalities than when they are presented to one modality alone (Bahrick \& Lickliter, 2000; Bahrick, Flom, \& Lickliter, 2002). For example, Bahrick, Flom, and Lickliter (2002) showed that 3-month-old infants are able to detect a change in tempo of a tapping toy hammer during redundant audiovisual but not unimodal (auditory or visual) presentations.

Studies with bobwhite quail chicks have also demonstrated the phenomenon of intersensory facilitation. Lickliter, Bahrick, and Honeycutt $(2002,2004)$ found that quail embryos are able to learn a maternal call significantly faster and remember the call significantly longer when the call is synchronized with a light (multimodal exposure), thereby providing intersensory redundancy, than when the call is presented alone (unimodal exposure). Associative learning studies have also shown similar results with infant rats. A study by Kucharski and Spear (1985) provided infant rats oral mixtures of a coffee and sucrose solution either simultaneously or consecutively (separated by 3 minutes) with an injection of lithium chloride (which produces effects similar to visceral illness). Preweanlings provided with the simultaneous compound solution ingested less 
sucrose during testing than those preweanlings who were given coffee and sucrose sequentially or given sucrose alone. These findings demonstrate enhancement of learning with simultaneous conditioned-stimulus presentations in contrast to successive CS presentations.

It is important to note that the IRH posits that it is the redundancy (i.e., the synchronously spatial and temporal occurrence of the same information across two or more senses) that recruits attention to the amodal properties, rather than simply the presence of more information overall. In fact, a number of studies with infants have demonstrated that asynchronous exposure disrupts learning of amodal properties such as rhythm (Bahrick \& Lickliter, 2000) and affect (Flom \& Bahrick, 2007). Bahrick, Castellanos, Argumosa (2011) also found that asynchronous stimulation disrupts speech perception. They assessed 4-month-old infants' ability to detect a change in meaning from passages that conveyed approval to passages that conveyed disapproval (or vice versa) by presenting them with either redundant audiovisual speech or nonredundant speech (i.e., unimodal auditory or asynchronous audiovisual). Infants presented with redundant audiovisual stimulation were able to detect the changes in prosody. In contrast, infants presented with nonredundant stimulation did not demonstrate detection of the prosody change. Similarly, studies with bobwhite quail have also shown that asynchronous stimulation can disrupt perception of amodal properties. Bobwhite quail chicks prenatally exposed to concurrent, asynchronous presentations of a bobwhite maternal call and patterned light were unable to demonstrate learning of the call in subsequent postnatal testing (Honeycutt \& Lickliter, 2001; Lickliter \& Hellewell, 1992). 
It is possible the asynchronous multimodal stimulus presentation results in a shift in attentional processes (Reynolds \& Lickliter, 2002).

\section{Unimodal Facilitation}

Unimodal facilitation has also been observed in research on early perceptual

development. For example, 2-month-old infants were able to discriminate faces based on modality-specific properties when the faces were presented unimodally, but not when presented with bimodal synchronous presentations (redundant audiovisual stimulation) (Bahrick, Lickliter, Vaillant, Schuman \& Castellanos, 2004). In addition, a study with 2month-old infants demonstrated that face-voice redundancy impairs face discrimination rather than merely the presence of stimulation in a second sense modality or a greater quantity of stimulation (Vaillant-Molina, Newell, Castellanos, Bahrick, \& Lickliter, 2006). A recent study by Castellanos (2012) found that nonredundant unimodal visual stimulation facilitates face identification (a modality-specific property) prior to the detection of prosody of speech (an amodal property). Further, 3- and 5-month old infants were able to detect a change in the direction or orientation, another modality-specific visual property, of a hammer tapping a surface when they were shown only a visual representation (unimodal stimulation) of the tapping hammer but not when they were shown an audiovisual (synchronous multimodal stimulation) video of the tapping hammer (Bahrick, Lickliter, \& Flom, 2006). An asynchronous condition was also conducted here to provide equivalent amounts and type of stimulation as the bimodal synchronous condition, however, no intersensory redundancy was available to compete for attention. Infants in this condition were also able to accurately discriminate the orientation of the hammer. Moreover, unimodal facilitation has been shown to occur in 4- 
year old children with memory for faces (Bahrick, Argumosa, Lopez, \& Todd, 2009). In this study, 4-year old children were shown female faces in unimodal visual presentations, asynchronous audiovisual presentations, or redundant audiovisual presentations under a brief familiarization period. They were then asked to discriminate between the familiarized female face and a novel female face. Face recognition was apparent in the unimodal and asynchronous audiovisual conditions, but not in the redundant audiovisual condition. The intersensory redundancy provided in the redundant audiovisual conditions presumably directed the infants' attention to the amodal properties of stimulation. These studies provide support for the second prediction of the IRH and indicate that modalityspecific properties are better detected in unimodal stimulation and attenuated in multimodal stimulation, where salient redundant amodal properties compete for infants' limited attention.

\section{Perceptual Improvements Across Development}

As young organisms acquire experience with their environment, they develop better efficiency and greater flexibility in their perceptual abilities. Hence, the third prediction of the IRH suggests that as individuals develop, they become better able to switch their attention between amodal and modality-specific properties in unimodal and multimodal events. The enhanced ability to pick up information more efficiently and the greater expertise of events and their properties results in less attentional resources necessary for processing information that went undetected in earlier development. Flom and Bahrick (2010) observed that 5-month old infants are able to detect and remember the orientation of a toy hammer tapping (a modality-specific visual property) only when it was presented in unimodal visual presentations (and not in bimodal audiovisual 
presentations) after a one-month retention interval. However, 9-month old infants could discriminate and remember the orientation of the hammer following the one-month retention interval when it was presented both unimodally and bimodally. In the social domain, Flom and Bahrick (2007) found that 4-month olds can detect affect only when speech is presented both bimodally and synchronously. In contrast, 5-month old infants could discriminate affect in synchronous audiovisual speech and in unimodal auditory speech, and 7-month olds were able to detect affect in all three conditions (i.e., bimodally, unimodal auditory speech, and unimodal visual speech).

\section{Facilitation Across Development}

The fourth prediction of the IRH states the conditions under which intersensory and unimodal facilitation will occur later in development. The ability to detect amodal and modality-specific properties in different events should depend on the relative familiarity and expertise the perceiver has with the task. That is, when the perceiver finds a task to be relatively difficult, more attentional resources will be required to perform the task. Therefore, this prediction posits that adults should experience intersensory and unimodal facilitation when a task is challenging relative to their familiarity or skill with the task. Bahrick and Lickliter (2004) found that 5-month old infants could discriminate simple tempo changes under both unimodal and bimodal presentations. However, Bahrick, Lickliter, Castellanos, and Vaillant-Molina (2010) increased the difficulty of the task by presenting the same aged infants with a more challenging tempo discrimination task. They found that 5-month olds showed intersensory facilitation as did 3-month old infants in the easier tempo discrimination task. In adult-based research, Santangelo and Spence (2007) and Santangelo, Ho, and Spence (2008) found that audiovisual and 
audiotactile cues better recruited spatial attention than did unimodal cues in tasks with more perceptually demanding stimuli. Participants discriminated the direction of visual targets after having seen either unimodal or bimodal cues under conditions of high or no perceptual load. Participants were able to discriminate the targets with unimodal and bimodal cues in the no-load condition. However, only the bimodal cues were able to recruit spatial attention in the high-load condition, again illustrating the role of multisensory information in directing attention in demanding or challenging situations. Hence, older perceivers should experience intersensory facilitation and unimodal facilitation across development when learning new information that is challenging relative to their skill level.

In summary, the IRH provides a model for how selective attention is distributed across stimulus conditions and across development on the basis of perceptual capabilities and experience. Intersensory redundancy results in the recruitment of attention to amodal properties of stimulation such as rhythm, tempo, and duration. In the absence of intersensory redundancy, attention is allowed to focus on modality-specific properties of events such as color, pitch, and face recognition. Hence, a number of extrinsic and intrinsic factors can affect the allocation of selective attention.

When exploring a multimodal event, attention is first distributed to the most salient (i.e., perceptually significant or relevant) features of stimulation, and is then distributed to less salient features. When attentional resources are limited or taxed, as is likely the case in infancy, attention to less salient properties may be disrupted altogether. However, as perceptual processing develops and the individual acquires more experience and familiarity with the properties of events, attention may be allocated more rapidly 
down the salience hierarchy, thereby allowing less salient properties to be detected and processed at a greater rate and for longer periods of time. This process results in attention being allocated to certain properties first, based on the presence or absence of intersensory redundancy, and then to other properties across exploratory time. The more attentional resources available and the more time available to explore the features of an event, the greater the amount of multimodal information processed. Therefore, understanding the mechanisms involved in driving selective attention across development is fundamental to understanding how and what is learned, which in turn, has implications for perception and cognition. 


\section{CHAPTER III}

\section{DETECTION OF MODALITY SPECIFIC PROPERTIES DURING PRENATAL}

\section{DEVELOPMENT}

Various postnatal studies with human infants have supported the second prediction of the IRH (i.e., unimodal facilitation) with the use of voices, faces, and orientation of objects. In order to investigate the role of selective attention in prenatal development, Vaillant (2010) designed a study to determine if prenatal unimodal exposure promotes learning of modality-specific properties of stimulation, and if prenatal redundant bimodal stimulation interferes with attention to and learning of modalityspecific properties of stimulation. Specifically, bobwhite quail chicks (Colinus virginianus) were exposed to either nonredundant unimodal (auditory only) exposure to a maternal call or redundant bimodal (audiovisual) exposure to a maternal call 24 hours prior to hatching. The chicks' postnatal auditory preferences between the familiarized call and the same call with altered pitch were assessed following hatching to determine if perceptual learning had occurred. It was hypothesized that the unimodally-exposed chicks would prefer the familiarized call over the pitch-modified call, whereas the bimodally-exposed chicks would not prefer the familiar call since the intersensory redundancy available during redundant audiovisual exposure would direct their attention to the amodal properties of stimulation.

To test these hypotheses, embryos were transferred to a sound attenuated stimulation room and placed in a portable hatcher approximately $24 \mathrm{hr}$ prior to hatching. The portable hatcher provided a transparent plastic window and a small opening, located directly above the embryos, upon which a lamp and speaker were placed to provide 
audiovisual presentations of a bobwhite maternal call. A computer software program ran an amplifier connected to the lamp that transmitted the pulsed light synchronously with the notes of the call.

\section{Procedure}

Bobwhite quail embryos were separated into 2 experimental conditions: a Unimodal Auditory group $(n=43)$ that was exposed to an individual variant of a bobwhite maternal call for $10 \mathrm{~min} / \mathrm{hr}$ during the $24 \mathrm{hr}$ prior to hatching, and a Bimodal Audiovisual group $(n=43)$ that was exposed to an individual variant of the bobwhite maternal call paired with a pulsing light temporally synchronized with the notes of the call for $10 \mathrm{~min} / \mathrm{hr}$ for $24 \mathrm{hr}$ prior to hatching. Another group of embryos served as controls $(n=28)$ and received no supplemental prenatal sensory stimulation. All subjects were then transferred to rearing tubs immediately after hatching and placed in groups of sameaged chicks until testing at $24 \mathrm{hr}$ following hatching.

Postnatal behavioral tests were conducted in a circular arena with two approach areas containing a small speaker attached to the arena wall. Two semi-circles representing each approach area, and comprising $5 \%$ of the total testing arena, were demarcated on a remote video monitor.

An original variant of the bobwhite maternal call was altered by modifying the pitch to one-step above and below the original pitch. Hence, two different modified calls were used for this experiment. All other acoustic features of the call remained constant. Pitch was altered by the use of computer multimedia software, MAGIX Audio Studio 10 Deluxe. Chicks from both exposure conditions thus received a simultaneous choice test 
between the same two maternal calls, but one call had the familiarized pitch and the other call had a novel pitch.

Testing involved placing each chick in the arena midway between the two approach areas. All birds were given a 5-min simultaneous choice test between the two variants of the same bobwhite maternal call. Each call originated from one of the speakers located in each of the two approach areas.

Results

Results are shown in Table 1 and 2. Results revealed that when quail embryos are not exposed to any prenatal sensory stimulation (Naïve Control group) they do not prefer $\left(\chi^{2}=1.357, p=.507\right)$ either variant of the bobwhite maternal call during testing $24 \mathrm{hr}$ after hatching. The naïve group also did not show significant differences in latency of approach or in the amount of time spent near either call. However, when quail embryos are prenatally exposed to auditory (unimodal) presentations of a maternal call they significantly prefer the familiarized call over a modified call with altered pitch at testing $24 \mathrm{hr}$ after hatching $\left(\chi^{2}=20.42, p<.00004\right)$. Wilcoxon signed-ranks test revealed that the unimodal group showed significantly longer duration $(\mathrm{z}=-3.526, \mathrm{p}=.000)$ and shorter latency $(\mathrm{z}=-2.789, \mathrm{p}=.005)$ scores for the familiarized call than the unfamiliarized call. In contrast, subjects that received prenatal audiovisual (bimodal) exposure to a maternal call failed to demonstrate a preference for either of the call variants at testing $24 \mathrm{hr}$ after hatching $\left(\chi^{2}=0.326, p>0.85\right)$. The bimodal group also showed no significant differences in their duration to the two calls $(z=-.700, p=.484)$ or latency $(\mathrm{z}=-.543, \mathrm{p}=.587)$ scores. Duration and latency scores were also converted into proportion of total duration time (PTDT) and proportion of total time to approach 
(PTTA) scores (duration/latency for the familiar call divided by total duration/latency for both calls). A one-way ANOVA demonstrated that the unimodal group had significantly larger PTD $(F(1,84)=12.148, p=.001)$ and lower latency $(\mathrm{PTTA})$ scores $(\mathrm{F}(1,84)=$ $4.310, \mathrm{p}=.041)$ to the familiar call than the bimodal group. The mean PTDT for both experimental conditions are shown in Figure 1.

\section{Table 1}

Means and Standard Deviations of Duration Scores (in seconds)

\begin{tabular}{|c|c|c|c|}
\hline $\begin{array}{l}\text { Stimulus } \\
\text { Condition } \\
\end{array}$ & $\mathbf{N}$ & Familiar Call & $\begin{array}{c}\text { Pitch-Modified } \\
\text { Call }\end{array}$ \\
\hline Naive & 28 & $\begin{array}{c}49.20 \\
(56.19)\end{array}$ & $\begin{array}{c}67.59 \\
(70.59)\end{array}$ \\
\hline $\begin{array}{l}\text { Nonredundant } \\
\text { Unimodal } \\
\text { Auditory }\end{array}$ & 43 & $\begin{array}{l}83.54 * \\
(62.82)\end{array}$ & $\begin{array}{c}35.45 \\
(47.59)\end{array}$ \\
\hline $\begin{array}{l}\text { Redundant } \\
\text { Audiovisual }\end{array}$ & 43 & $\begin{array}{c}54.05 \\
(58.03)\end{array}$ & $\begin{array}{c}59.42 \\
(53.37)\end{array}$ \\
\hline
\end{tabular}




\section{Table 2}

Means and Standard Deviations of Latency Scores (in seconds)

\begin{tabular}{cccc}
\hline $\begin{array}{c}\text { Stimulus } \\
\text { Condition }\end{array}$ & $\mathbf{N}$ & Familiar Call & $\begin{array}{c}\text { Pitch-Modified } \\
\text { Call }\end{array}$ \\
\hline Naive & 28 & 90.08 & 67.75 \\
& & $(92.29)$ & $(87.17)$ \\
Nonredundant & & \\
Unimodal \\
Auditory & 43 & $57.62 *$ & 119.31 \\
Redundant & & $(52.28)$ & $(111.58)$ \\
Audiovisual & & 67.96 \\
& 43 & 60.07 & $(80.17)$ \\
\hline $\mathrm{p}<.05$ (Wilcoxon Test) & & $(73.55)$ &
\end{tabular}

Figure 1

Mean Proportion of Total Duration Time for the Familiar Call

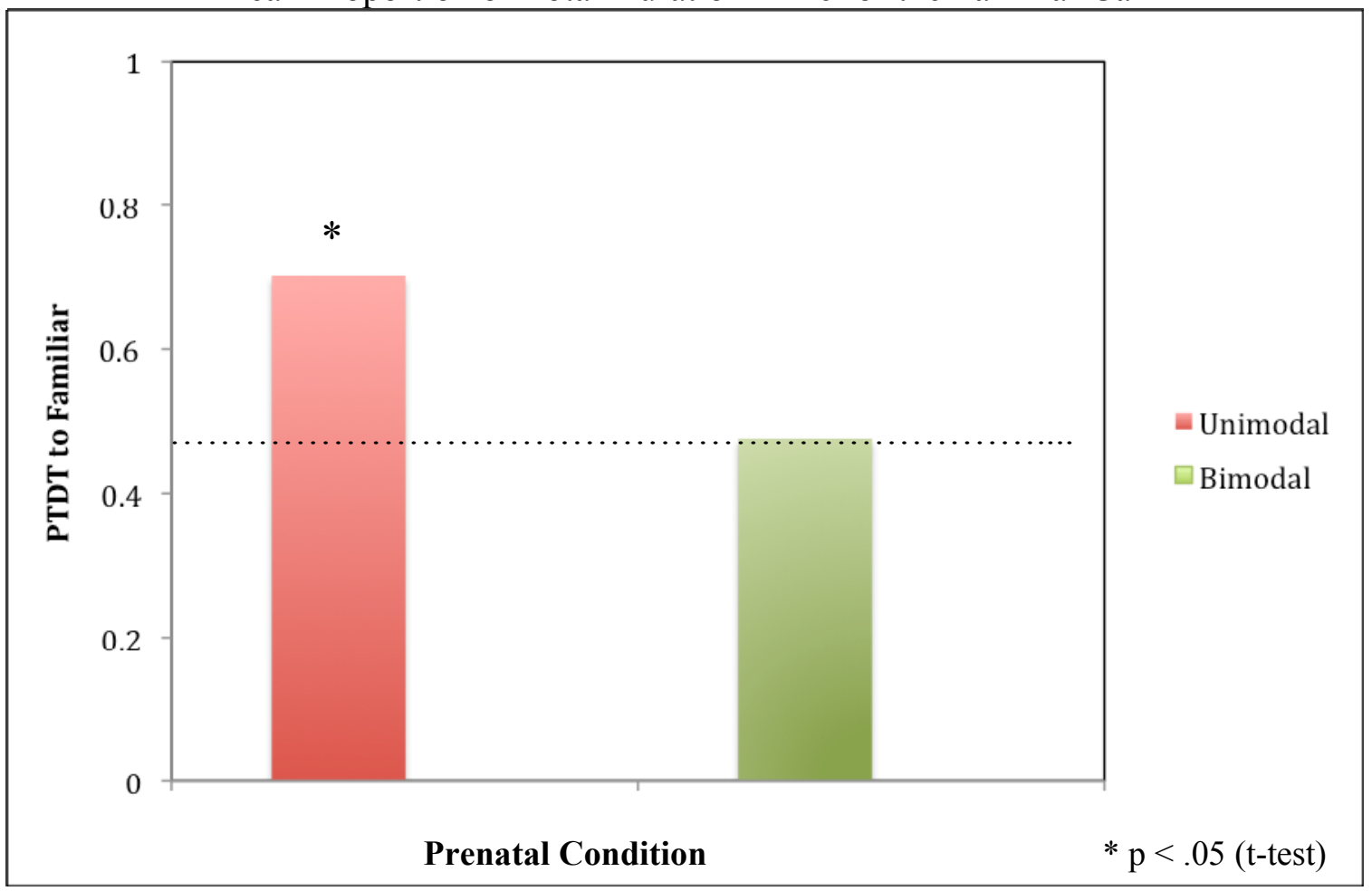




\section{Summary}

The purpose of this study was to investigate the second prediction of the Intersensory Redundancy Hypothesis, which predicts that early in development information presented to one sense modality facilitates attention to the modality-specific properties of that information and facilitates perceptual learning of those properties at the expense of amodal properties. The IRH posits that during unimodal stimulation there is no information presented redundantly and thus competing for attention. Therefore, this lack of redundancy allows the organism to focus on and process the modality-specific properties of stimulation present in the information. The results of this prenatal study supported this prediction by demonstrating that prenatal unimodal exposure facilitates learning of modality-specific properties of stimulation, whereas redundant bimodal stimulation interferes with attention to and learning of modality-specific properties of stimulation. Chicks prenatally exposed to auditory presentations of a bobwhite maternal call significantly preferred that maternal call over a pitch-modified call. In contrast, chicks prenatally exposed to synchronous audiovisual presentations of the maternal call did not prefer the familiar call over the pitch-modified call, indicating no detection of the pitch change. The IRH suggests that detection of the pitch change, as seen with the unimodally-exposed chicks, is a consequence of the chicks' selective attention to the modality-specific property of pitch during prenatal exposure. Hence, these findings indicate that when intersensory redundancy is not available to compete for attention, modality-specific properties can be attended to and processed successfully, even during prenatal development. 
The present study demonstrated the usefulness of comparative studies using precocial birds to investigate developmental mechanisms and processes involved in the development of intersensory perception. Studies with human subjects are limited in the extent to which experimental manipulations of sensory experience can be made.

Comparative animal research allows experimental alterations of sensory experience, such as prenatal sensory augmentation, deprivation, and displacement in order to explore organismic and environmental factors underlying early perceptual development. 


\section{CHAPTER IV}

\section{RESEARCH DESIGN}

The current study was designed to address the third and fourth predictions of the Intersensory Redundancy Hypothesis. Specifically, the third prediction posits that as development proceeds, attention becomes more efficient, flexible, and better organized, thereby allowing for the detection of both amodal and modality-specific information in both redundant, multimodal and nonredundant, unimodal stimulation. Further, the fourth prediction predicts that when task difficulty is high and attentional resources are exhausted, intersensory and unimodal facilitation occur across the lifespan.

On the basis of our research (Vaillant, 2010), which demonstrated that quail embryos were able to detect changes in pitch only when exposed prenatally to unimodal presentations of a maternal call, the current study investigated if both unimodal and synchronous bimodal presentations administered later in development (postnatally) serve as a basis for learning a modality-specific property of stimulation (see Chapter III). As previously mentioned, studies using human subjects have supported this prediction (Flom \& Bahrick, 2007, 2010).

In the current study, unimodal and bimodal perception during early postnatal development was explored in bobwhite quail chicks. Prior experiments demonstrated that chicks prenatally exposed to a maternal call were only able to detect a pitch change in subsequent postnatal testing when they were exposed to unimodal (auditory only) presentations of the call. Embryos exposed to bimodal (redundant auditory-visual) presentations were unable to detect a pitch change in subsequent postnatal tests. The present study provided the same stimulus presentations and tests. However, the chicks 
were of an older developmental age, with more perceptual experience, thereby making the test seemingly easier for the subject. According to the IRH, if the chicks' perceptual abilities, processing efficiency, and flexibility of attention have increased sufficiently to reduce the overall difficulty of the task for the chicks, then chicks should be able to detect the pitch change under both nonredundant, unimodal and redundant, bimodal stimulation. It was therefore hypothesized that both the unimodal auditory exposure and the bimodal audiovisual exposure would allow for the detection and processing of the modalityspecific property of pitch.

Experiment 1 tested this hypothesis by exposing bobwhite quail chicks to an individual bobwhite maternal call either redundantly (synchronous audio-visual stimulation) or non-redundantly (unimodal auditory or asynchronous audio-visual stimulation) approximately one day after hatching. According to the IRH, intersensory facilitation is due to redundancy and not simply due to the greater overall amount of stimulation in two modalities compared to one modality alone. Consistent with this view, various studies have found that intersensory facilitation is not observed under conditions of multimodal stimulation without redundancy (e.g., asynchronous but equivalent visual and auditory patterns, Bahrick \& Lickliter, 2000; Bahrick, Flom, \& Lickliter, 2002; Flom \& Bahrick, 2007; Lickliter, Bahrick, \& Honeycutt, 2002). Hence, an asynchronous bimodal condition was included here in which congruent but asynchronous visual and auditory stimulation were provided. Twenty-four hours after the completion of the auditory or audiovisual stimulation, chicks were tested individually between the familiar version of the maternal call that was presented previously (i.e., no acoustic changes) 
versus the same maternal call with an altered pitch range (all other acoustic features held constant).

The fourth prediction of the IRH proposes that if the task difficulty is increased (relative to the experience of the perceiver), then unimodal facilitation will be apparent. Thus, it was hypothesized that if the difficulty of the task has increased sufficiently relative to the expertise of the bobwhite chick (by narrowing the pitch range between the familiarized maternal call and the novel pitch-modified call), attentional resources will be more taxed and unimodal facilitation should occur. Chicks receiving redundant, bimodal exposure would have their attention focused on amodal stimulus properties such as rhythm or duration of the call, and as such, should not detect the pitch change during subsequent testing. Specifically, unimodal auditory exposure would facilitate attention to and learning of the modality-specific property of pitch, whereas redundant audiovisual exposure would interfere with learning of the modality-specific property. It was also hypothesized that the asynchronous audiovisual exposure would facilitate attention to and learning of the modality-specific property of pitch because there would be no redundancy competing for attention.

Experiment 2 thus tested the fourth prediction of the IRH. Specifically, chicks were exposed to a maternal call under unimodal auditory, synchronous audiovisual, or asynchronous audiovisual conditions approximately one day after hatching. Twenty-four hours after the completion of the stimulus presentation, chicks were tested individually between the familiar version of the call presented previously versus the same call with an altered pitch range (all other acoustic features held constant). However, the range in the 
pitch between the familiar and the pitch-modified call was narrowed with respect to that of the first experiment. This was done to increase the overall difficulty of the task.

\section{Hypotheses}

The following research questions were addressed in this study (predicted results are shown in Tables 3 and 4):

Experiment 1: Having acquired further experience with auditory stimuli and developed better perceptual capabilities by $24 \mathrm{hr}$ of age, will the unimodal facilitation effect become less evident in young chicks' perceptual learning?

Hypothesis: Chicks postnatally exposed to auditory only, asynchronous audiovisual, and redundant audiovisual presentations of the bobwhite maternal call will prefer the familiar call over the pitch-modified call, thereby indicating detection of the pitch change. Due to the low level of difficulty in this task, relative to the expertise of the perceiver, chicks will not require that the information be presented unimodally or asynchronously in order to attend to and process the modality-specific property of pitch.

Experiment 2: When same-aged chicks (24 hr of age) are presented a challenging task, relative to the level of difficulty familiarity with that task, will the unimodal facilitation effect become evident across early postnatal development?

Hypothesis 1: Narrowing the pitch range between the familiarized call and the pitch-modified call will increase the difficulty of the task. In this more difficult task, chicks postnatally exposed to auditory only and asynchronous audiovisual presentations of the bobwhite maternal call will prefer the familiar call over the pitch-modified call in subsequent tests, thereby indicating detection of the pitch change. 
Hypothesis 2: In contrast, same-aged chicks postnatally exposed to redundant audiovisual presentations will not prefer the familiar call over the pitch-modified call, thereby indicating no detection of the pitch change. Because of the relative difficulty of the task, chicks will require that the information be presented unimodally or asynchronously (i.e., no redundancy) in order to attend to and process the modalityspecific property of pitch (unimodal facilitation). According to the IRH, when a task is perceived as relatively challenging to the perceiver, intersensory redundancy will direct attention toward amodal properties of stimulation. Hence, in this redundant audiovisual condition, I predict chicks will detect and process the amodal properties of the call (tempo, rhythm) rather than the modality-specific properties (pitch).

\section{Table 3}

Hypotheses for Experiment 1

\begin{tabular}{ccc}
\hline \multicolumn{1}{c}{ Condition } & Stimulation & Expected Preference \\
\hline Nonredundant Unimodal & Auditory only & Familiar Call \\
Nonredundant Bimodal & Asynchronous Audiovisual & Familiar Call \\
Redundant Bimodal & Synchronous Audiovisual & Familiar Call \\
\hline
\end{tabular}

Predicted results for chicks' preference as a function of stimulus condition (nonredundant unimodal auditory, nonredundant audiovisual, redundant audiovisual) 


\section{Table 4}

Hypotheses for Experiment 2

\begin{tabular}{ccc}
\hline Condition & Stimulation & Expected Preference \\
\hline Nonredundant Unimodal & Auditory only & Familiar Call \\
Nonredundant Bimodal & Asynchronous Audiovisual & Familiar Call \\
Redundant Bimodal & Synchronous Audiovisual & No Preference \\
\hline
\end{tabular}

Predicted results for chicks' preference as a function of stimulus condition (nonredundant unimodal auditory, nonredundant audiovisual, redundant audiovisual) 


\section{CHAPTER V}

\section{GENERAL METHOD}

\section{Subjects}

Subjects were 227 incubator reared bobwhite quail chicks (Colinus virginianus). Fertilized unincubated eggs were received weekly from a commercial supplier and set in an incubator maintained at $75-80 \%$ relative humidity and $37.5^{\circ} \mathrm{C}$. Embryonic age was calculated on the basis of the first day of incubation as Day 0, and so forth. To control for possible variations in developmental age, only those birds that hatched on Day 23 were used as subjects. Following hatching, groups of 10-12 subjects were housed in a rearing tub until testing. Chicks were given constant access to food and water, except during testing sessions. Ambient air temperature was maintained at approximately $30^{\circ} \mathrm{C}$ both in the rearing and testing rooms.

\section{Apparatus}

Approximately $24 \mathrm{hrs}$ after hatching, chicks were transferred to a sound attenuated stimulation room, maintained at approximately $30^{\circ} \mathrm{C}$, and placed in a tub with food and water covered with a metal wire net. Audio-visual stimulus presentations were delivered by means of a computer with a custom designed software program running a speaker that broadcasted the maternal call. The speaker was placed on an exterior side of the plastic tub. The computer software program also ran an amplifier connected to an adjustable lamp that transmitted the pulsed light synchronously with the notes of the call. The lamp was placed directly above the tub and visible to the chicks. In this condition, the synchronized light provided the same amodal information (onset, rhythm, rate, and duration) as the five notes of the maternal call. Hence, chicks were exposed to stimulus 
presentations at $24 \mathrm{hr}$ of age (approximately $48 \mathrm{hr}$ later than exposure time in the prenatal study).

Behavioral tests were conducted with chicks at $72 \mathrm{hr}$ of age in an arena $130 \mathrm{~cm}$ in diameter, encircled by a wall $60 \mathrm{~cm}$ in height. The arena surface was painted white, and an opaque white curtain covered the wall of the arena. A video camera mounted directly above the arena allowed for remote observation and data collection. Two semi-circular approach areas each comprising approximately $5 \%$ of the total area of the testing arena were demarcated on a remote video monitor used for data gathering (see Figure 2). Both approach areas contain a small speaker mounted to the arena wall and hidden behind the white curtain to allow for the presentation of auditory stimuli during the testing trials.

Figure 2

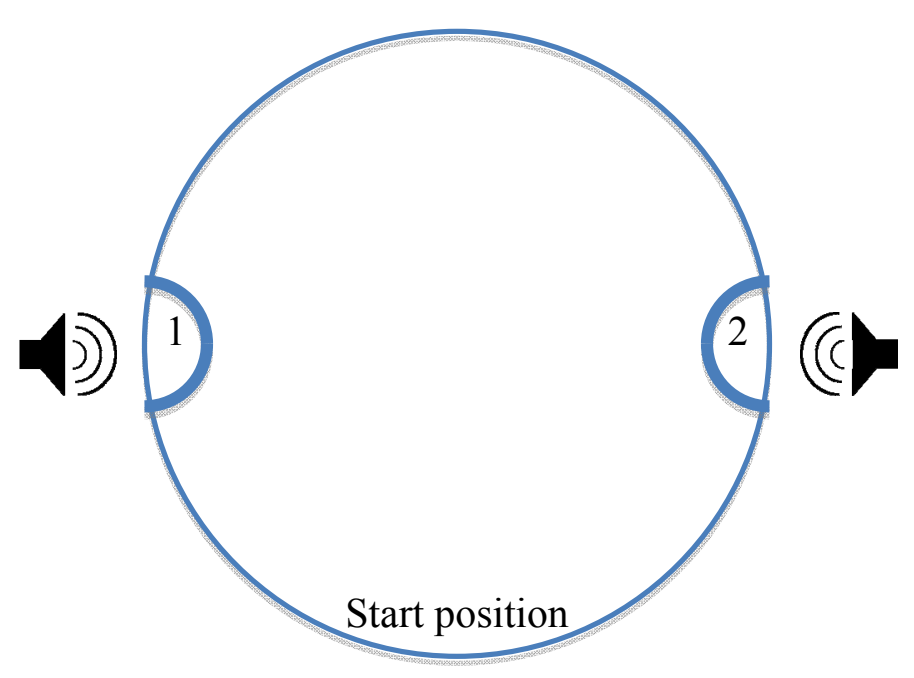

View of the Behavioral Testing Arena. Approach areas 1 and 2 broadcasted either the familiar call or the pitch-modified call. Call location was counterbalanced across testing trials. 


\section{Procedure}

Bobwhite quail chicks were divided into 3 experimental conditions: (1) a Unimodal Auditory group was exposed to an individual variant of the bobwhite maternal assembly call for $10 \mathrm{~min} / \mathrm{hr}$ for $24 \mathrm{hr}$ starting at approximately $24 \mathrm{hr}$ following hatching (48 hr later than exposure time in the prenatal study), (2) a Redundant Bimodal Audiovisual group was exposed to an individual variant of the bobwhite maternal call paired with a pulsing light temporally synchronized with the notes of the call for 10 min/hr for $24 \mathrm{hr}$, and (3) an Asynchronous Bimodal Audiovisual group was exposed to the same maternal call concurrently with the pulsing light presented out of synchrony with the notes of the call (offset by $300 \mathrm{~ms}$ ) for $24 \mathrm{hr}$, thereby providing audiovisual stimulation with no intersensory redundancy. A modified version of the computer software Javascript was used to create the stimulation schedules. Pitch of the maternal call was altered by the use of computer multimedia software, MAGIX Audio Studio 10 Deluxe.

Testing was conducted $24 \mathrm{hr}$ following stimulation completion (at $72 \mathrm{hr}$ following hatching) and consisted of a 5 min (300s) simultaneous choice test. All subjects were tested individually between the familiar version of the maternal call that was presented previously versus the same maternal call altered to a higher or lower pitch range (all other acoustic features held constant). Hence, chicks from all exposure conditions were given a simultaneous choice test between the same two bobwhite maternal calls, but one call retained its familiarized pitch and the other call had a novel pitch. 


\section{Data Analyses}

Nonparametric tests were conducted along with parametric tests, which evaluated between-group differences. However, it was expected that results of the parametric and nonparametric tests would not necessarily correspond because of the high level of variability in such interval data. In order for a group to be considered as having detected the modality-specific property of pitch, at least one parametric and one nonparametric test had to reveal statistical significance. Groups that did not meet this standard were considered to have shown no significant preference (i.e., no detection of the pitch change).

The data of interest for the parametric analyses were measures of duration (in seconds) for the auditory stimuli presented during testing. A proportion of total duration time (PTDT) was calculated from the time a chick spent in the approach area containing the familiar version of the maternal call relative to the total duration spent in both familiar and modified approach areas. A proportion of .50 represents chance responding. In contrast, a proportion greater than .50 reflects a majority of time spent in the approach area containing the familiar version of the maternal call, whereas a proportion less than .50 represents a majority of time spent in the approach area containing the modified call. One-sample $t$ tests were used to evaluate whether the PTDT spent in the modified call approach area was significantly greater than chance. A One-Way Analysis of Variance (ANOVA) was used to evaluate between group comparisons of PTDT to the familiar call, and Tukey's HSD was used for multiple comparison analyses.

Nonparametric analyses were conducted on the duration of time spent in each approach area by subjects in a group using the Wilxocon Signed-Ranks Test. 
Significance levels of $\mathrm{p}<.05$ (two-tailed) were used to evaluate all results. Latency of initial approach to the familiar and pitch-modified calls was also recorded for each subject. Latency data, however, were found to be highly variable across subjects and thus will not be discussed in the individual experiments. 


\section{CHAPTER VI}

\section{EXPERIMENT 1}

\section{Developmental Enhancement of Selective Attention (Easy Discrimination Task)}

Prior experiments have demonstrated that unimodal presentations of a maternal call provided prenatally facilitate learning of the modality-specific property of pitch (Vaillant, 2010). In this previous study, bobwhite quail embryos were exposed to either unimodal (auditory only) or bimodal (synchronous audiovisual) presentations of a maternal call $24 \mathrm{hr}$ prior to hatching. Chicks at $24 \mathrm{hr}$ of age were then tested in a simultaneous choice between the familiarized version of the maternal call presented prenatally versus the same maternal call with altered pitch. Only the chicks prenatally exposed to unimodal presentations showed a significant preference for the familiarized version of the maternal call in subsequent testing, thereby demonstrating detection of the pitch change. These results are in line with the IRH prediction suggesting that modalityspecific properties are best detected under unimodal conditions, where redundancy is not available and therefore not directing attention toward amodal properties. However, the IRH also predicts that as individuals develop, processing becomes more efficient, attention becomes more flexible, and perceptual differentiation increases. These developmental changes in perception should allow for the detection of amodal and modality-specific properties in both nonredundant unimodal and redundant bimodal stimulation.

This experiment aimed to investigate this latter prediction in light of previous findings. Therefore, the same procedure and pitch ranges (i.e., one-step lower and higher pitch ranges from the original maternal call) were used for this experiment as were used 
in the prenatal study (Vaillant, 2010). However, bobwhite quail were now exposed postnatally to a maternal call and tested at a later developmental age between the familiarized version of the maternal call versus the same call with altered pitch. It was hypothesized that chicks, having more developed perceptual systems at this later stage in development, would find this task relatively easier than it had been earlier in development, and thus, chicks should detect pitch changes under both Nonredundant Unimodal and Redundant Bimodal stimulus conditions. A third group received Nonredudant Bimodal (asynchronous audiovisual) presentations of the maternal call. This group was expected to detect the pitch change in subsequent testing as well, since no redundancy was present to direct attention to amodal properties of the maternal call.

\section{Method}

One hundred and sixteen bobwhite quail chicks, divided into 3 experimental conditions (Unimodal, Redundant Bimodal, and Asynchronous Bimodal) served as subjects. All chicks were exposed in groups of 10-12 to an individual maternal call altered to a one-step lower or higher pitch range (all other acoustic features held constant) for $10 \mathrm{~min}$ each hour for a $24 \mathrm{hr}$ period starting at approximately $24 \mathrm{hr}$ following hatching. All groups were exposed to the same maternal call altered to a one-step lower or higher pitch range (all other acoustic features held constant). The Unimodal group (N $=41)$ received only auditory stimulation, the Redundant Bimodal group $(\mathrm{N}=35)$ were exposed to the same call synchronously paired with a pulsing light (intersensory redundancy), while the Nonredundant Bimodal group $(\mathrm{N}=40)$ received the same call concurrently with the pulsing light presented out of synchrony with the notes of the call (no redundancy). For each condition, 50\% of subjects received exposure to the maternal 
call altered to a one-step higher pitch, and $50 \%$ of subjects received the maternal call altered to a one-step lower pitch. All groups were tested 24 hours after the completion of the stimulation ( $72 \mathrm{hr}$ of age) in a simultaneous choice test between the two individual variants of the maternal call (a full two-step range). Hence, exposure and testing utilized the same stimulation and presentation protocol that was provided previously in our prenatal study (Vaillant, 2010).

Results and Discussion

Results are shown in Table 5. Wilcoxon signed-ranks test revealed that the Unimodal group showed significantly longer duration $(z=-4.024, p=.000)$ scores for the familiar call than the pitch-modified call. Subjects in the Redundant Bimodal group also showed significantly longer duration $(\mathrm{z}=-4.259, \mathrm{p}=.000)$ scores for the familiar call. Subjects in the Nonredundant Bimodal group likewise demonstrated significantly longer duration to the familiar call $(\mathrm{z}=-2.863, \mathrm{p}=.004)$.

One-sample $t$ tests were performed on the proportion of total duration time (PTDT) spent in the approach area with the familiarized version of the call against the chance value of .50 for all experimental groups. Results revealed that chicks exposed to Unimodal presentations of a maternal call showed a greater PTDT to the familiar version of the call at testing $(t(40)=6.007, \mathrm{p}=.000)$. Similarly, chicks exposed to Redundant Bimodal presentations of the call showed a greater PTDT to the familiar version of the call at testing $(t(34)=6.328, \mathrm{p}=.000)$, as did chicks in the Nonredundant Bimodal group $(t(39)=2.924, \mathrm{p}=.006)$. Mean PTDT scores for each condition are shown in Table 6. A one-way analysis of variance compared the mean PTDT to the familiar call for all experimental groups. This test was not found to be statistically significant at an alpha 
level of $.05(F(2,113)=3.015, \mathrm{p}=.053)$. A Tukey HSD test indicated that the mean PTDT to the familiar call for the Unimodal group $(\mathrm{M}=.753, \mathrm{SD}=.269)$ was not significantly greater than the mean PTDT for the Nonredundant Bimodal group ( $\mathrm{M}=$ $.626, \mathrm{SD}=.272)$ or the Redundant Bimodal group $(\mathrm{M}=.748, \mathrm{SD}=.232)$. The mean PTDT for the Redundant Bimodal group also did not differ significantly from the mean PTDT for the Nonredundant Bimodal group.

\section{Table 5}

Means and Standard Deviations of Duration Scores (in seconds) for Experiment 1

\begin{tabular}{cccc}
\hline $\begin{array}{c}\text { Stimulus } \\
\text { Condition }\end{array}$ & $\mathbf{N}$ & Familiar Call & $\begin{array}{c}\text { Pitch-Modified } \\
\text { Call }\end{array}$ \\
\hline $\begin{array}{c}\text { Nonredundant } \\
\text { Unimodal } \\
\text { Auditory }\end{array}$ & 41 & $97.81^{*}$ & 31.00 \\
Nonredundant & & $(71.46)$ & $(46.47)$ \\
Audiovisual & 40 & $69.65^{*}$ & \\
& & $(54.81)$ & $(29.47)$ \\
Redundant & & & \\
Audiovisual & 35 & $89.69^{*}$ & 26.78 \\
\hline $\mathrm{*} p<.05$ (Wilcoxon Test) & & $(56.71)$ & $(27.42)$ \\
\hline
\end{tabular}




\section{Table 6}

Mean Proportion of Total Duration Time and Standard Deviations (in seconds) for the Familiar Call For Experiment 1

\begin{tabular}{ccc}
\hline Stimulus Condition & Mean PTDT & SD \\
\hline $\begin{array}{c}\text { Nonredundant Unimodal } \\
\text { Auditory }\end{array}$ & $.7527^{*}$ & .269 \\
Nonredundant Audiovisual & $.6259^{*}$ & .272 \\
& & .232 \\
\hline Redundant Audiovisual & $.7476^{*}$ & \\
\hline
\end{tabular}

$$
* \mathrm{p}<.05(t \text {-test })
$$

Consistent with the predictions of the Intersensory Redundancy Hypothesis, I hypothesized that chicks receiving unimodal (auditory only) exposure, redundant bimodal exposure, and asynchronous bimodal exposure to a maternal call would all prefer that familiarized call over the same call with altered pitch. Since quail chicks have previously been shown to detect this pitch change when exposed to the call prenatally, having acquired more experience and flexible perceptual capabilities across development, I predicted they should be able to detect the change in the modality-specific property of pitch under both non-redundant and redundant exposure conditions. Results revealed that chicks in the nonredundant Unimodal and Asynchronous conditions and the redundant Bimodal condition were able to detect a pitch change in subsequent testing between the familiar version of the call and the pitch-modified call. 
These findings support the third prediction of the IRH, which states that as individuals develop and gain experience with the features of the environment, their perceptual and learning capabilities will increase. Thus, the need for unimodal stimulus presentations in order to detect and attend to modality-specific properties of stimulation becomes less evident. Chicks prenatally exposed to a bobwhite maternal call were able to detect a pitch change in subsequent postnatal testing only when they were provided with prenatal unimodal presentations. In contrast, chicks postnatally exposed to a bobwhite maternal call were able to detect this pitch change after having received nonredundant unimodal or redundant bimodal stimulus presentations. The chicks' ability to detect this pitch change signifies that the task had become easier relative to their perceptual capabilities. However, if a more difficult discrimination task is presented, will the effect of unimodal facilitation become evident again? The following experiment was designed to address this question. 


\section{CHAPTER VII}

\section{EXPERIMENT 2}

\section{Unimodal Facilitation under Increasing Difficulty (Difficult Discrimination Task)}

According to the Intersensory Redundancy Hypothesis, continued experience with the properties of events fosters perceptual differentiation such that more salient properties are processed first while less salient properties necessitate extended processing time (Bahrick \& Lickliter, 2012). It is likely that this perceptual differentiation results in faster detection of previously differentiated properties in subsequent exposures, leading to more flexible shifts in attention among familiar event properties. Therefore, the degree of unimodal/intersensory facilitation observed in an individual should depend on the level of familiarity and/or difficulty with the task relative to the expertise of the perceiver. As processing efficiency and attentional systems improve across development, unimodal and intersensory facilitation should become less apparent. However, if tasks are made difficult enough to challenge the perceiver, unimodal and intersensory facilitation should again become evident, even in experienced perceivers.

Experiment 2 was designed to explore unimodal facilitation under conditions of higher task difficulty. Here, the same design from the first experiment (i.e., postnatal exposure and subsequent testing) was used, however, the simultaneous choice test was made more difficult by narrowing the pitch range used between the familiar version of the maternal call and the pitch-modified maternal call. It was hypothesized that the nonredundant Unimodal and Asynchronous Bimodal groups would detect the pitch change by demonstrating a preference for the familiar version of the call. However, the Redundant Bimodal group was expected not to detect the pitch change (i.e., show no 
preference), since the relative difficulty of the task would result in selective attention to the redundantly specified amodal properties of the call.

Method

A total of 111 bobwhite quail chicks, divided into 3 experimental conditions (Unimodal, Redundant Bimodal, and Asynchronous Bimodal) served as subjects. All chicks were exposed in groups of 10-12 to an individual maternal call and tested between two variants of the call as in Experiment 1. However, in this experiment, the range between the pitch of the call presented previously and the pitch of the call with which they were subsequently tested was narrowed, thereby increasing the difficulty of the discrimination task. Specifically, chicks were tested between an original variant of the bobwhite maternal call and the same maternal call with either a one-step higher pitch or a one-step lower pitch. For each condition, $50 \%$ of subjects received exposure to the maternal call altered to a one-step higher pitch or one-step lower pitch, and $50 \%$ of subjects received the standard maternal call (no pitch modification). Hence, the pitch variation between the two calls here varied by one full step, while the pitch variation in Experiment 1 varied by two full steps. The Unimodal group $(\mathrm{N}=39)$ received only auditory stimulation, the Redundant Bimodal $(\mathrm{N}=35)$ received temporally synchronized audiovisual stimulation, and the Nonredundant Bimodal group $(\mathrm{N}=37)$ received asynchronous audiovisual stimulation. This condition served to control for the possibility that the Bimodal condition interfered with the detection of the modality-specific property simply because it provided more overall stimulation than the Unimodal condition. All groups were tested 24 hours after the completion of the exposure ( $72 \mathrm{hr}$ of age) in a 
simultaneous choice test between the familiarized and modified variants of the bobwhite maternal call (a one-step range, compared to the two-step range used in Experiment 1).

\section{Results and Discussion}

Results are shown in Table 7. Wilcoxon signed-ranks test revealed that the Unimodal group showed significantly longer duration $(z=-2.986, p=.003)$ scores for the familiar call than the pitch-modified call. Chicks in the Nonredundant Bimodal group also showed significantly longer duration $(\mathrm{z}=-2.950, \mathrm{p}=.003)$ to the familiar call. In contrast, chicks in the Redundant Bimodal group showed no significant differences in their duration $(\mathrm{z}=-.950, \mathrm{p}=.342)$ scores for the two variants of the call.

One-sample $t$ tests were performed on the proportion of total duration time (PTDT) spent in the approach area with the familiarized version of the call against the chance value of .50 for all experimental groups. Results revealed that chicks exposed to Unimodal presentations of a maternal call showed a greater PTDT to the familiar version of the call at testing $(t(38)=3.316, \mathrm{p}=.002)$, as did chicks in the Nonredundant Bimodal group, $(t(36)=2.968, \mathrm{p}=.005)$. In contrast, chicks exposed to Redundant Bimodal presentations of the call did not show greater PTDT to the familiar version of the maternal call at testing $(t(34)=.683, \mathrm{p}=.499)$. Mean PTDT scores for each condition are shown in Table 8 .

A one-way analysis of variance compared the mean PTDT to the familiar call of all experimental groups. The comparison test was not found to be statistically significant at an alpha level of $.05,(\mathrm{~F}(2,108)=1.683, \mathrm{p}=.191)$. A Tukey HSD test indicated that the mean PTDT to the familiar call for the Unimodal group $(\mathrm{M}=.643, \mathrm{SD}=.270)$ was not significantly greater than the mean PTDT for the Nonredundant Bimodal group ( $\mathrm{M}=$ 
$.624, \mathrm{SD}=.253)$ or the Redundant Bimodal group $(\mathrm{M}=.534, \mathrm{SD}=.291)$. The mean

PTDT for the Redundant Bimodal group also did not differ significantly from the mean

PTDT for the Nonredundant Bimodal group.

\section{Table 7}

Means and Standard Deviations of Duration Scores (in seconds) for Experiment 2

\begin{tabular}{|c|c|c|c|}
\hline $\begin{array}{l}\text { Stimulus } \\
\text { Condition }\end{array}$ & $\mathbf{N}$ & Familiar Call & $\begin{array}{c}\text { Pitch-Modified } \\
\text { Call }\end{array}$ \\
\hline $\begin{array}{l}\text { Nonredundant } \\
\text { Unimodal Auditory }\end{array}$ & 39 & $\begin{array}{l}72.46^{*} \\
(52.90)\end{array}$ & $\begin{array}{c}40.33 \\
(48.92)\end{array}$ \\
\hline $\begin{array}{l}\text { Nonredundant } \\
\text { Audiovisual }\end{array}$ & 37 & $\begin{array}{l}70.82 * \\
(49.43)\end{array}$ & $\begin{array}{c}35.75 \\
(24.75)\end{array}$ \\
\hline $\begin{array}{l}\text { Redundant } \\
\text { Audiovisual }\end{array}$ & 35 & $\begin{array}{c}57.05 \\
(50.50)\end{array}$ & $\begin{array}{c}53.97 \\
(59.28)\end{array}$ \\
\hline
\end{tabular}

$* \mathrm{p}<.05$ (Wilcoxon Test)

Table 8

Mean Proportion of Total Duration Time and Standard Deviations (in seconds) for the Familiar Call For Experiment 2

\begin{tabular}{ccc}
\hline Stimulus Condition & Mean PTDT & SD \\
\hline $\begin{array}{l}\text { Nonredundant Unimodal } \\
\text { Auditory }\end{array}$ & $.6433^{*}$ & .270 \\
Nonredundant Audiovisual & $.6235^{*}$ & .253 \\
Redundant Audiovisual & .5335 & .291 \\
\hline$* \mathrm{p}<.05(t$-test $)$ & & \\
\hline
\end{tabular}


Despite the non-significant differences between groups in their PTDT scores (as indicated in the ANOVA results), individual group analyses demonstrated that chicks receiving nonredudant unimodal (auditory only) and asynchronous bimodal (audiovisual) exposure to a maternal call preferred that call over the same call with altered pitch. In contrast, chicks receiving redundant bimodal (audio-visual synchrony) exposure did not prefer that call over the same call with altered pitch. When the pitch range was narrowed so that the detection of the pitch change became more difficult relative to the perceptual capabilities of the perceiver, unimodal facilitation was observed. Specifically, chicks in the Unimodal and the Nonredundant Bimodal groups were able to detect the pitch change between the familiar and pitch-modified versions of the call. On the other hand, chicks in the Redundant Bimodal group did not demonstrate detection of the pitch change. These results are consistent with the predictions of the IRH. That is, when the task was made challenging relative to the skill level of the perceiver, unimodal facilitation was evident, since chicks that received nonredundant, unimodal stimulation were able to detect and process modality-specific properties (in this case, pitch of the maternal call). 


\section{CHAPTER VIII}

\section{GENERAL DISCUSSION}

Previous research has shown that bobwhite quail chicks prenatally exposed to unimodal (auditory only) presentations of a maternal call were able to detect a pitch change in subsequent postnatal testing (at $24 \mathrm{hr}$ of age) between the familiar version of the call and a pitch-modified call; however, prenatal redundant bimodal presentations of the maternal call did not facilitate detection of the pitch change, providing evidence of unimodal facilitation and redundant interference with detection of modality-specific properties of sensory stimulation (Vaillant, 2010). In Experiment 1 of the present study, chicks were exposed postnatally to the maternal call $24 \mathrm{hr}$ following hatching and subsequently tested at $72 \mathrm{hr}$ of age in a choice test between the familiar version of the call versus a pitch-modified call (the same two variants of the maternal call used in the prenatal study). Chicks from both redundant and nonredundant experimental groups were able to detect the pitch change in subsequent testing. These findings demonstrate a developmental shift in perceptual learning capabilities from prenatal to the period following hatching.

The results are consistent with the Intersensory Redundancy Hypothesis Bahrick \& Lickliter, 2000, 2002), which predicts that older perceivers should develop more flexible perceptual capabilities with prior experience, thereby allowing these perceivers to detect and attend to amodal and modality-specific properties under both redundant and nonredundant stimulus conditions. In other words, improved processing abilities and increased experience with events facilitates detection and processing of information not detected at an earlier time in development. 
Research has shown that infants' perceptual capabilities shift from recognition of global to more specific, local information (Fricke, Colombo, \& Allen, 2000) and from detection of information about object function to more detailed information about an object's form and structure (Oakes \& Madole, 2008; Xu, Carey, \& Quint, 2004). The notion of a shift in perceptual skills is in line with E.J. Gibson's concept of increasing specificity, which suggests that individuals initially differentiate more global information and then progressively differentiate more detailed and precise features of stimulation with increasing perceptual experience (Gibson, 1969).

Research has shown that infants' attentional and perceptual abilities improve and become more malleable across development (e.g., Bahrick, Lickliter, Vaillant, Shuman, \& Castellanos, 2004; Frick, Colombo, \& Saxon, 1999; Mayes \& Kessen, 1989; Shaddy \& Colombo, 2004). For example, infants' perception and discrimination of modalityspecific properties have been shown to improve in studies exploring face and voice recognition. Bahrick, Lickliter, Vaillant, Shuman \& Castellanos (2004a) found that 2month old infants demonstrate unimodal facilitation for face identification. In contrast, 3month old infants were able to discriminate faces in both nonredundant unimodal and redundant audiovisual stimulation (Bahrick, Lickliter, Vaillant, Shuman, \& Castellanos, 2004b). Similarly, Bahrick, Lickliter, Shuman, Batista, Castellanos \& Newell (2005) demonstrated that infants initially discriminate voices in only nonredundant unimodal stimulation, but later in development are also able to do so in redundant audiovisual stimulation. Together with the current study's results, these findings demonstrate improved detection and processing of modality-specific properties of stimulation across development. 
Across the lifespan, individuals regularly engage in perceptual tasks that are challenging relative to their prior experience or familiarity with that task. For example, adults are typically remarkably proficient in discriminating human faces. However, having considerably less experience with other primates, for instance, it is likely that discriminating chimpanzee faces would be a rather difficult task in comparison. Learning a new language would also be a relatively challenging task. According to the IRH, when individuals encounter tasks that are difficult or challenging relative to their skill level, unimodal and intersensory facilitation effects should again become evident, even in later stages of development.

Experiment 2 explored this prediction by examining the detection of pitch within a more challenging task compared to Experiment 1 . The pitch range between the bobwhite maternal call presented $24 \mathrm{hr}$ after hatching and the maternal call against which it was tested was narrowed. The pitch modification generated a more subtle change in pitch (compared to the pitch range used in the first experiment) between the variants of the maternal call used in testing. Specifically, chicks were exposed postnatally to a maternal call $24 \mathrm{hr}$ following hatching and subsequently tested at $72 \mathrm{hr}$ of age in a choice test between the familiar version of the call versus a pitch-modified call (as in the first experiment). However, the choice test between the two maternal calls was made more difficult by decreasing the pitch range between the calls by one full step. In this second experiment, the effects of unimodal facilitation were apparent. Chicks from the nonredundant unimodal and nonredundant (asynchronous) audiovisual groups were able to detect the pitch change during testing. However, chicks exposed to redundant audiovisual stimulation of the maternal call did not detect the pitch change during 
subsequent testing. These results are consistent with the fourth prediction of the IRH, indicating that unimodal facilitation should become evident across development during tasks that are relatively challenging to the perceiver. In instances of high task difficulty or cognitive load, attention will be directed toward the more salient features at the expense of less salient features. In other words, in difficult tasks, the intersensory redundancy provided in audiovisual stimulation will direct attention toward amodal properties, while modality-specific properties would become perceptual background. Hence, in the redundant audiovisual condition in Experiment 2, intersensory interference resulted in attention being directed toward the amodal properties (e.g., rhythm, duration, tempo) of the maternal call rather than the modality-specific property of pitch.

As mentioned previously, research has shown that under challenging or demanding conditions, infant and adult performance on such tasks can revert to earlier stages of development in which unimodal and intersensory facilitation are evident. For example, research indicates that adults' discrimination of tempo provides evidence of intersensory facilitation during high task difficulty (Bahrick, Todd, Argumosa, Grossman, Castellanos, \& Sorondo, 2009). In this study, adults were exposed to either unimodal visual or synchronous audiovisual presentations of a toy hammer tapping at a standard tempo (amodal property). They were then tested with various trials depicting no change in tempo, a $25 \%$ change (low difficulty), a $17 \%$ change (moderate difficulty), or a $9 \%$ change in tempo (high diffulty). Participants had to report whether the tempo of each trial was the same or different from the familiarized tempo. Results revealed the task was difficult overall for adults, evidenced by the low correct response rate. More importantly, results showed that more correct responses were reported for the redundant audiovisual 
condition than the unimodal visual condition, thereby demonstrating intersensory facilitation, as was seen with infants in prior studies (Bahrick, Lickliter, Castellanos, \& Vaillant-Molina, 2010). Similarly, chicks in Experiment 2 of the current study demonstrated the same pattern as younger subjects had in the prenatal study, in which unimodally-exposed chicks preferred the familiar call versus the pitch-modified call while bimodally-exposed chicks did not. Findings such as these suggest important implications for perception and learning across the lifespan.

Research now indicates that the effects of unimodal facilitation are apparent across species, across stimulus conditions, and across developmental time points, thereby demonstrating the generalizability of this effect. The results of the current study provide new information about the nature of selective attention across early development, specifically, the apparent changes in performance that occur as a result of experience and enhanced perceptual capabilities. Selective attention and perceptual processing provide a foundation for what is learned and remembered, and thus, studies like the one presented here are fundamental to understanding the underlying developmental trajectories of perceptual development.

\section{Limitations}

The current study used pre-recorded auditory presentations of a bobwhite maternal call along with a flashing light emanating from a lamp synchronized with the notes of the call. The bimodal stimulation allowed for a high level of experimental control in generating redundant, synchronous audiovisual stimulus presentations as well as asynchronous stimulus presentations. Although multimodal information is undoubtedly provided in the natural environment, these types of audiovisual stimulus 
combinations are not typically encountered in natural settings. More ecologically valid bimodal presentations could use synchronized vestibular and auditory stimulation, resembling the more natural vibratory sensations produced from vocalizations of the mother hen. Nevertheless, the effect of unimodal facilitation was evident here despite having used non-ecologically valid stimuli. This effect displays the generalizability of the developmental processes proposed by the IRH across different stimulus conditions. Another concern to note is the standard laboratory conditions employed in the current study. The chick's natural environment is comprised of complex arrays and amounts of stimulation, including predators, weather changes, and natural objects not encountered in the laboratory setting. The quail chicks used in this study, in comparison, were restricted in terms of available information provided before and after hatching. Aside from their conspecifics and the controlled sensory stimulation provided, there was limited information provided for the chicks. Therefore, whether pitch detection is feasible or even relevant in a quail chick's natural environment remains to be determined.

Nevertheless, all events provide modality-specific information that is important to the understanding of the event. Calls and songs are critical communicative tools for avian species. For example, bobwhite quail are known to use calls for the purpose of maintaining and regrouping pairs and coveys, particularly important for defense against predators (Johnsgard, 1974). Other avian species are also known to use vocalizations for various functions. For example, white-throated sparrows (Zonotrichia albicollis) use specific note frequencies in the birds' vocalizations for identification of their conspecifics (Hurly, Ratcliffe, Weary, \& Weisman, 1992). Black-capped chickadees (Poecile atricapillus provide another example of pitch perception in birds. In this species, males 
generate a certain internote pitch change to attract and stimulate females (Weisman \& Ratcliffe, 2004). Thus, the use and detection of changes in vocalizations are important skills for birds and appear to develop early in life.

\section{Future Directions}

The Intersensory Redundancy Hypothesis has been demonstrated to be a useful model for investigating the types of intersensory relations organisms are expected to detect at certain points in development. It can also address particular mechanisms that may underlie the development of specific intersensory skills. Specifically, it can provide further insight into the role of selective attention as a mechanism for facilitating perceptual processing, ultimately influencing what individuals learn and remember. Future studies can further explore the differences underlying the perception of amodal feature relations versus the perception of modality-specific feature relations in order to understand how the two processes work together to integrate and perceive multimodal objects and events.

\section{Summary}

The Intersensory Redundany Hypothesis posits that amodal properties are likely more salient than modality-specific properties in early development due to the redundancy available in our multimodal environment. However, for animals and humans alike, detection and processing of modality-specific properties of stimulation are also fundamental to perception and learning. For example, detection of pitch and timbre, (modality-specific properties) allow a person to discriminate between voices of different individuals. Color and visual patterns are critical to distinguishing between different objects or persons. Efficient social engagement necessitates the ability to recognize and 
relate individual faces with individual voices. Therefore, the perception of amodal and modality-specific properties is necessary for typical social and cognitive development.

The current study provides insight into bobwhite quail chicks' attentional and perceptual changes across early development. Specifically, it provides clues as to how detection of specific stimulus properties can change as an individual develops. How perceptual learning develops with experience is fundamental to understanding processes of typical and atypical cognitive and social development. Research investigating early patterns of selective attention can provide further insight into a range of developmental outcomes. Perceptual development contributes to language, social, and cognitive development; therefore, research on the mechanisms of selective attention and perceptual processing of events can lead to a better understanding of and intervention strategies for populations with attentional and processing impariments (e.g., attention deficit disorder, autism spectrum disorders, brain damage/injury).

A final note to mention is the role of animal models in perceptual and developmental research. Bobwhite quail chicks are precocial animals that have functioning auditory and visual systems even prior to hatching. Their development also progresses at a rather rapid rate in comparison to human infants. This rapid developmental trajectory in perceptual capabilities observed in quail chicks emphasizes the usefulness of such animal models for research in perceptual development. Despite the differences across species, comparative research has been shown to be useful for investigating the role of selective attention in early perceptual development. For example, Lickliter, Bahrick, and Markham (2006) presented bobwhite quail embryos initially with redundant bimodal stimulation and then subsequently with nonredundant unimodal 
stimulation. Chicks demonstrated a significant preference for the familiar call over a novel maternal call in a choice test 2 days after hatching. Chicks that had received prenatal unimodal presentations or prenatal unimodal-first, bimodal-second presentations of the maternal call showed no preference for the familiarized call in postnatal testing. These results showed that exposure to amodal properties in bimodal stimulation (such as rhythm, tempo, duration) can educate attention to amodal properties in subsequent unimodal stimulation during prenatal development. Studies such as these, along with the one presented here, provide support for the use of comparative research for investigating perceptual development. 


\section{REFERENCES}

Adler, S.A., Gerhardstein, P., \& Rovee-Collier, C. (1998). Levels-of-processing effects in infant memory? Child Development, 69, 280-94.

Bahrick, L.E. (1983). Infants' perception of substance and temporal synchrony in multimodal events. Infant Behavior \& Development, 6, 429-451.

Bahrick, L.E. (1987). Infants' intermodal perception of two levels of temporal structure in natural events. Infant Behavior and Development, 10, 387-416.

Bahrick, L.E. (1988). Intermodal learning in infancy: Learning on the basis of two kinds of invariant relations in audible and visible events. Child Development, 59, $197-$ 209.

Bahrick, L.E. (1992). Infants' perceptual differentiation of amodal and modality specific audiovisual relations. Journal of Experimental Child Psychology, 53, 180-199.

Bahrick, L.E. (1994). The development of infants' sensitivity to arbitrary intermodal relations. Ecological Psychology, 6, 111-123.

Bahrick, L.E. (2001). Increasing specificity in perceptual development: Infants' detection of nested levels of multimodal stimulation. Journal of Experimental Child Psychology, 79, 253-270.

Bahrick, L.E., Argumosa, M., Lopez, H., \& Todd, J.T. (2009, October). Face discrimination in preschool-age children. Poster presented at the Cognitive Developmental Society meeting, San Antonio, TX.

Bahrick, L.E., Castellanos, I., Argumosa, M.A. (2011). Intersensory redundancy facilitates infants' perception of meaning in speech passages. Manuscript in preparation.

Bahrick, L.E., Flom, R., \& Lickliter, R. (2002). Intersensory redundancy facilitates discrimination of tempo in 3-month-old infants. Developmental Psychobiology, $41,352-363$.

Bahrick, L.E., \& Lickliter, R. (2000). Intersensory redundancy guides attentional selectivity and perceptual learning in infancy. Developmental Psychology, 36, 190-201.

Bahrick, L.E., \& Lickliter, R. (2002). Intersensory redundancy guides early cognitive and perceptual development. In R. Kail \& H. Reese (Eds.), Advances in child development and behavior (Vol. 30, pp. 153-187). New York: Academic Press. 
Bahrick, L.E., \& Lickliter, R. (2004). Infants' perception of rhythm and tempo in unimodal and multimodal stimulation: A developmental test of the intersensory redundancy hypothesis. Cognitive, Affective and Behavioral Neuroscience, 4, 137-147.

Bahrick, L.E., \& Lickliter, R. (2012). The role of intersensory redundancy in early perceptual, cognitive, and social development. In: A. Bremner, D. J. Lewkowicz, \& C. Spence (Eds.), Multisensory development. Oxford University Press.

Bahrick, L.E., Lickliter, R., Castellanos, I., \& Vaillant-Molina. M. (2010). Intersensory redundancy and tempo discrimination in infancy: The roles of task difficulty and expertise. Developmental Science, 13, 731-737.

Bahrick, L.E., Lickliter, R., \& Flom, R. (2006). Up versus down: The role of intersensory redundancy in the development of infants' sensitivity to the orientation of moving objects. Infancy, 9, 73-96.

Bahrick, L.E., Lickliter, R., Shuman, M.A., Batista, L.C., Castellanos, I., \& Newell, L. (2005, November). The development of infant voice discrimination: From unimodal auditory to bimodal audiovisual stimulation. Poster presented at the International Society of Developmental Psychobiology, Washington, D.C.

Bahrick, L.E., Lickliter, R., Vaillant, M., Shuman, M.A., \& Castellanos, I. (2004a, May). Infant discrimination of faces in the context of dynamic, multimodal events: Predictions from the intersensory redundancy hypothesis. Poster presented at the International Conference on Infant Studies, Chicago, IL.

Bahrick, L.E., Lickliter, R., Vaillant, M., Shuman, M.A., \& Castellanos, I. (2004b, June). The development of face perception in dynamic, multimodal events: Predictions from the intersensory redundancy hypothesis. Poster presented at the International Multisensory Research Forum, Barcelona, Spain.

Bahrick, L.E., \& Newell, L.C. (2008). Infant discrimination of faces in naturalistic events: actions are more salient than faces. Developmental Psychology, 44, 983996.

Bahrick, L.E., \& Pickens, J. (1994). Amodal relations: The basis for intermodal perception and learning in infancy. In D. J. Lewkowicz \& R. Lickliter (Eds.), The development of intersensory perception: Comparative perspectives (pp. 205-233). Hillsdale, NJ: Erlbaum.

Bahrick, L.E., Todd, J.T., Argumosa, M.A., Grossman, R., Castellanos, I., \& Sorondo, 
B.M. (2009, July). Intersensory facilitation across the life-span: Adults show enhanced discrimination of tempo in bimodal vs. unimodal stimulation. Presented at the International Multisensory Research Forum, New York, NY.

Bahrick, L.E., Walker, A.S., and Neisser, U. (1981). Selective looking by infants. Cognitive Psychology, 13, 377-390.

Bremner, A.J., Lewkowicz, D.J., \& Spence, C. (in press). Multisensory Development. Oxford, UK: Oxford University Press.

Castellanos, I. (2011). Infants' selective attention to faces and prosody of speech: The roles of intersensory redundancy and exploratory time. FIU Electronic Theses and Dissertations. Paper 526.

Flom, R., \& Bahrick, L.E. (2007). The development of infant discrimination of affect in multimodal and unimodal stimulation: The role of intersensory redundancy. Developmental Psychology, 43, 238-252.

Flom, R., \& Bahrick, L.E. (2010). The effects of intersensory redundancy on attention and memory: Infants' long-term memory for orientation in audiovisual events. Developmental Psychology, 46, 428-436.

Frick, J. E., Colombo, J., \& Allen, J. R. (2000). Temporal sequence of global-local processing in 3-month-old infants. Infancy, 1, 375-386.

Frick, J. E., Colombo, J., \& Saxon, T. F. (1999). Individual and developmental differences in disengagement of fixation in early infancy. Child Development, 70, 537-548.

Gibson, E.J. (1969). Principles of perceptual learning. New York: Appleton-CenturyCrofts.

Gibson, E.J. (1988). Exploratory behavior in the development of perceiving, acting, and the acquiring of knowledge. Annual Review of Psychology, 39, 1-41.

Gibson, E.J. \& Pick, A.D. (2000). An ecological approach to perceptual learning and development. Oxford University Press.

Gibson, J.J. (1966). The senses considered as perceptual systems. Boston: HoughtonMifflin.

Gibson, J.J. (1979). The ecological approach to visual perception. Boston: HoughtonMifflin.

Gottlieb, G. (1971). Ontogenesis of sensory function in birds and mammals. In E. 
Tobach, L. R. Aronson, \& E. Shaw (Eds.), The biopsychology of development (pp. 67-128). New York: Academic Press.

Gottlieb, G. (1993). Social induction of malleability in ducklings: Sensory basis and psychological mechanism. Animal Behaviour, 45, 707-719.

Honeycutt, H., \& Lickliter, R. (2001). Order-dependent timing of unimodal and multimodal stimulation affects prenatal auditory learning in bobwhite quail embryos. Developmental Psychobiology, 38, 1-10.

Honeycutt, H., \& Lickliter, R. (2002). Prenatal experience and postnatal perceptual preferences: Evidence for attentional-bias in bobwhite quail embryos (Colinus virginianus). Journal of Comparative Psychology, 116, 270-276.

Hurly, T.A., Ratcliffe, L., Weary, D.M., \& Weisman, R. (1992). White-throated sparrows (Zonotrichia albicollis) can perceive pitch change in conspecific song by using the frequency ration independent of the frequency difference. Journal of Comparative Psychology, 106(4), 388-391.

Jaime, M., Bahrick, L.E., \& Lickliter, R. (2010). The critical role of temporal synchrony in the salience of intersensory redundancy during prenatal development. Infancy, $15,61-82$.

Jiang, W., Jiang, H., \& Stein, B.E. (2002). Two corticotectal areas facilitate multisensory orientation behavior. Journal of Cognitive Neuroscience, 14, 1240-1255.

Johnsgard, P. A. (1974). Quail music: The complex calls of a bird contain clues to its evolution. Natural History, 83(3), 34-40.

Koch, C. \& Ullman, S. (1985). Shifts in selective visual attention: Towards the underlying neural circuitry. Human Neurobiology 4, 219-227.

Kucharski, D., \& Spear, N. E. (1985). Potentiation and overshadowing in preweanling and adult rats. Journal of Experimental Psychology: Animal Behavior Processes, $11,15-34$.

Lewkowicz, D.J. (1988a). Sensory dominance in infants: 1. Six-month-old infants' response to auditory-visual compounds. Developmental Psychology, 24, 155171.

Lewkowicz, D.J. (1988b). Sensory dominance in infants: 2. Ten-month-old infants' response to auditory-visual compounds. Developmental Psychology, 24, 172182.

Lewkowicz, D.J. (1996). Infants' response to the audible and visible properties of the 
human face: Role of lexical syntactic content, temporal synchrony, gender, and manner of speech. Developmental Psychology, 32, 347-366.

Lewkowicz, D.J. (2000). The development of intersensory temporal perception: An epigenetic systems/limitations view. Psychological Bulletin, 126, 281-308.

Lewkowicz, D.J., \& Lickliter, R. (1994a). The development of intersensory perception: Comparative perspectives. Hillsdale, NJ: Erlbaum.

Lewkowicz, D.J., \& Lickliter, R. (1994b). Insights into mechanisms of intersensory development: The value of a comparative, convergent-operations approach. In D. J. Lewkowicz \& R. Lickliter (Eds.), The development of intersensory perception: Comparative perspectives (pp. 403-413). Hillsdale, NJ: Erlbaum.

Lewkowicz, D.J., \& Turkewitz, G. (1980). Cross-modal equivalence in early infancy: Auditory-visual intensity matching. Developmental Psychology, 16, 597-607.

Lickliter, R., \& Bahrick, L.E. (2000). The development of infant intersensory perception: Advantages of a comparative, convergent-operations approach. Psychological Bulletin, 126, 260-280.

Lickliter, R., Bahrick, L.E., \& Honeycutt, H. (2002). Intersensory redundancy facilitates perceptual learning in bobwhite quail embryos. Developmental Psychology, 38, $15-23$.

Lickliter, R., Bahrick, L.E., \& Honeycutt, H. (2004). Intersensory redundancy enhances memory in bobwhite quail embryos. Infancy, 5, 253-269.

Lickliter, R., Bahrick, L.E., \& Markham, R.G. (2006). Intersensory redundancy educates selective attention in bobwhite quail embryos. Developmental Science, 9, 605616.

Lickliter, R., \& Hellewell, T. (1992). Contextual determinants of auditory learning in bobwhite quail embryos and hatchlings. Developmental Psychobiology, 25, 1-24.

Lickliter, R., \& Lewkowicz, D. J. (1995). Intersensory experience and early perceptual development: Attenuated prenatal sensory stimulation affects postnatal auditory and visual responsiveness in bobwhite quail chicks (Colinus virginianus). Developmental Psychology, 31, 609-618.

Lickliter, R., \& Stoumbos, J. (1991). Enhanced prenatal auditory experience facilitates species-specific visual responsiveness in bobwhite quail chicks (Colinus virginianus). Journal of Comparative Psychology, 105, 89-94.

Mayes, L., \& Kessen, W. (1989). Maturational changes in measures of habituation. Infant 
Behavior and Development, 12, 437-450.

Oakes, L. M., \& Madole, K. L. (2008). Function revisited: How infants construe functional features in their representation of objects. In R. Kail (Ed.). Advances in child development and behavior, vol 36 (pp. 135-185). San Diego: Elsevier.

Reynolds, G.D. \& Lickliter, R. (2002). Effects of prenatal sensory stimulation on heart rate and behavioral measures of arousal in bobwhite quail embryos. Developmental Psychobiology, 41, 112-122.

Santangelo, V., Ho, C., \& Spence, C. (2008). Capturing spatial attention with multisensory cues. Psychonomic Bulletin and Review, 15, 398-403.

Santangelo, V., \& Spence, C. (2007). Multisensory cues capture spatial attention regardless of perceptual load. Journal of Experimental Psychology: Human Perception and Performance, 33, 1311-1321.

Shaddy, D. J., \& Colombo, J. (2004). Developmental changes in infant attention to dynamic and static stimuli. Infancy, 5, 355-365.

Stein, B.E., Meredith, M.A., Honeycutt, W.S., \& McDade, L. (1989). Behavioral indices of multisensory integration: Orientation to visual cues is affected by auditory stimuli. Journal of Cognitive Neuroscience, 1, 12-24.

Vaillant, J. (2010). Detection of modality-specific properties during prenatal development in unimodal and bimodal events. FIU Electronic Theses and Dissertations. Paper 303.

Vaillant-Molina, M., Newell, L., Castellanos, I., Bahrick, L.E., \& Lickliter, R. (2006, June). Intersensory redundancy impairs face perception in early development. Presented at the International Conference for Infant Studies, Kyoto, Japan.

Walker-Andrews, A.S., Bahrick, L.E., Raglioni, S.S. \& Diaz, I. (1991). Infants' bimodal perception of gender. Ecological Psychology, 3, 55-75.

Weisman, R., Ratcliffe, L. (2004). Relative pitch and the song of black-capped chickadees. American Scientist, 92, 532-539.

Xu, F., Carey, S., \& Quint, N. (2004). The emergence of kind-based object individuation in infancy. Cognitive Psychology, 49, 155-190. 
VITA

\section{JIMENA VAILLANT-MEKRAS}

2005

2007-2012

2010

2010-2012
B.S., Psychology

University of Florida

Gainesville, Florida

MBRS Research Initiative for Scientific Enhancement

Fellowship

Funded by the National Institute of Health (NIH)

M.S., Psychology

Florida International University

Miami, Florida

Doctoral Candidate

Florida International University

Miami, Florida

\section{PUBLICATIONS AND PRESENTATIONS}

Pham-Escobar, B., Vaillant, J., \& Lickliter, R. (2007, November). Modified heart rate interferes with perceptual learning in bobwhite quail embryos. Poster presented at the International Society for Developmental Psychobiology, San Diego, CA.

Vaillant, J. (2010). Detection of modality-specific properties during prenatal development in unimodal and bimodal events. FIU Electronic Theses and Dissertations. Paper 303.

Vaillant, J., Bahrick, L.E., \& Lickliter, R. (2008, November). Detection of modalityspecific properties in unimodal and bimodal events. Poster presented at the International Society for Developmental Psychobiology, Washington, D.C.

Vaillant, J., Bahrick, L.E., \& Lickliter, R. (2009, March). Detection of modality-specific properties is enhanced by unimodal exposure during prenatal development. Poster presented at the Society for Research in Child Development, Denver, CO.

Vaillant, J., Bahrick, L.E., \& Lickliter, R. (2011, November). Testing the intersensory redundancy hypothesis during early development. Poster presented at the International Society for Developmental Psychobiology, Washington, D.C.

Vaillant, J. \&, Lickliter, R. (2010, November). Selective attention during prenatal 
development: Redundancy across auditory and vibro-tactile stimulation facilitates learning in quail embryos. Research presented at the FIU MBRS Mini-Symposium, Miami, FL.

Vaillant, J. \&, Lickliter, R., Pham-Escobar, B. (2007, September). Modified heart rate interferes with perceptual learning in bobwhite quail embryos. Research presented at the FIU MBRS Mini-Symposium, Miami, FL. 